\title{
A Novel Linguistic Z-Number QUALIFLEX Method and Its Application to Large Group Emergency Decision Making
}

\author{
Xue-Feng Ding (iD, Li-Xia Zhu, Mei-Shun Lu, Qi Wang, and Yi-Qi Feng \\ School of Management, Shanghai University, Shanghai 200444, China \\ Correspondence should be addressed to Xue-Feng Ding; athena_tju@sina.com
}

Received 24 November 2019; Revised 17 March 2020; Accepted 10 June 2020; Published 26 June 2020

Academic Editor: Rahman Ali

Copyright (C) 2020 Xue-Feng Ding et al. This is an open access article distributed under the Creative Commons Attribution License, which permits unrestricted use, distribution, and reproduction in any medium, provided the original work is properly cited.

\begin{abstract}
After an unconventional emergency event occurs, a reasonable and effective emergency decision should be made within a short time period. In the emergency decision making process, decision makers' opinions are often uncertain and imprecise, and determining the optimal solution to respond to an emergency event is a complex group decision making problem. In this study, a novel large group emergency decision making method, called the linguistic Z-QUALIFLEX method, is developed by extending the QUALIFLEX method using linguistic $Z$-numbers. The evaluations of decision makers on the alternative solutions are first expressed as linguistic $Z$ numbers, and the group decision matrix is then constructed by aggregating the evaluations of all subgroups. The QUALIFLEX method is used to rank the alternative solutions for the unconventional emergency event. Besides, a real-life example of emergency decision making is presented, and a comparison with existing methods is performed to validate the effectiveness and practicability of the proposed method. The results show that the proposed linguistic Z-QUALIFLEX can accurately express the evaluations of the decision makers and obtain a more reasonable ranking result of solutions for emergency decision making.
\end{abstract}

\section{Introduction}

Emergency decision making is a human activity based on cognitive information, especially in the large group context. How to effectively express human cognitive information and perform cognitive computation in the large group emergency decision making is a highly challenging task and issue. Recently, a lot of studies have been carried out for cognition information representation and computation in solving various decision making problems, which include location evaluation $[1,2]$, enterprise resource planning system selection [3, 4], doctor selection [5], medical inquiry application evaluation [6], and others [7-9]. These research studies have proposed many effective methods and algorithms for using cognitive information in practical decision making processes. In this paper, we will pay attention to the large group emergency decision making problem based on human cognitive information.

Over the past several years, different unconventional emergency events occurred frequently all over the world.
Examples include the Indian Ocean tsunami in 2004, Hurricane Katrina in the US in 2005, the 9.0 magnitude earthquake in Japan in 2011, and the Tianjin Port explosion in China in 2015, among others. These emergency events are difficult to predict and often impossible to control. They not only bring painful disasters and huge losses of life and properties but also cause serious social panic and potential secondary derivative hazards. When an emergency event happens, to reduce casualties and property losses and to eliminate various potential derivative hazards, decisions of reasonable and effective emergency relief solutions need to be made within a short time period [10]. However, because of their complexity, inadequate precursors, and devastating and potential secondary derivatives, it is difficult to deal with unconventional emergency events by conventional emergency management methods [11]. Therefore, the emergency decision making problem has gained increasing attention from both scholars and practitioners over recent years [12-21]. 
Previous works in solving emergency decision making problems involve only a small number of decision makers. However, such problems are usually large group emergency decision making (LGEDM) problems. Emergency management often involves many different organizations and departments, and the decision making team often includes a large number of experts from various professional backgrounds [12]. The large team, or large group, decision making problems have the following characteristics [21, 22]: (a) the team may have 20 or more members, and the members cooperate but may also have conflicts; (b) the various decision criteria may be incommensurate although related; and (c) the opinions of the team members may change over time. The large group decision making problem aims to support decision makers in making the best choice from all the alternatives or solutions while considering multiple criteria effectively and efficiently. Therefore, it is preferred to address emergency decision making problems in the environment of the large group.

Owing to the uncertainty and complexity of the emergency situations, it is hard for decision makers to express their judgments with accuracy, especially under time pressure [23]. Various theories, such as fuzzy sets, intuitionistic fuzzy sets, and hesitant fuzzy sets, have been proposed to deal with imprecise decision information. However, these theories are inefficient in expressing the reliability of decision makers' assessments [24]. This study uses linguistic $Z$-numbers [6] to represent inaccurate judgments of the decision makers. Compared with other methods such as fuzzy sets, intuitionistic fuzzy sets, and hesitant fuzzy sets, the linguistic $Z$-numbers can not only describe the cognition of the decision makers better but also consider the reliability of the cognitive information so as to represent the evaluations of decision makers more accurately.

The QUALIFLEX (Qualitative Flexible Multiple Criteria) method was developed by Paelinck [25] for group decision making using cardinal and ordinal information under uncertainty and fuzzy circumstance. Compared with other outranking methods such as SAW (simple additive weighting method), WP (weighted product method), LA (liner allocation method), TOPSIS, and ELECTRE, the QUALIFLEX method is a more flexible sorting method. In the QUALIFLEX method, a concordance/discordance index is first determined for each pair of alternatives through pairwise comparisons of the alternatives with respect to each criterion for all possible permutations of the alternatives. A weighted concordance/discordance index is then calculated for each pair of alternatives in each permutation. Finally, a comprehensive concordance/discordance index is calculated for each possible permutation of the alternatives. The permutation with the maximal value of the comprehensive concordance/discordance index is determined to be the best permutation, and the alternative ranked on the top of the best permutation is identified as the optimal alternative [26].

In order to describe the decision maker's assessment more accurately under the emergency circumstance and make an effective emergency decision as quickly as possible, this study develops an extended QUALIFLEX method using linguistic $Z$-numbers, called the linguistic Z-QUALIFLEX method, for solving LGEDM problems. Linguistic $Z$-numbers are first used to represent the decision makers' evaluations of the alternative solutions over various criteria, and the decision makers are divided into several subgroups according to the similarities of their evaluations. A group linguistic decision matrix is then constructed by aggregating the evaluations of all subgroups. The QUALIFLEX method is finally used to rank the alternative emergency solutions. Besides, an empirical example is provided to verify the applicability and the effectiveness of the proposed method. The proposed linguistic Z-QUALIFLEX method can accurately express the evaluations of the decision makers, characterize the similarities of their evaluations, and can also help decision makers get the best solution quickly and effectively.

The remainder of this paper is organized as follows. Previous related works are briefly reviewed in Section 2. Section 3 briefly introduces the basic concepts of $Z$-numbers and the QUALIFLEX method. Section 4 presents the linguistic Z-QUALIFLEX method for LGEDM problems. In Section 5, a numerical example is presented, and a comparative analysis is provided to illustrate the feasibility and validity of the proposed method. Conclusions and suggestions for future research are presented in Section 6.

\section{Literature Review}

Nowadays, a variety of methods have been proposed for EDM in the literature. For example, Ding et al. [27] proposed a new EDM approach by using picture fuzzy sets and an axiomatic design technique for determining the optimal rescue plan to reduce the damages of emergencies. Ding and Liu [28] presented an integrated approach based on prospect theory and the VIKOR (VIseKriterijumska Optimizacija I Kompromisno Resenje) method for EDM with 2-dimension uncertain linguistic information, and Ding and Liu [29] solved the EDM problem by the use a combined approach of Pythagorean fuzzy uncertain linguistic variables and zerosum game theory. $\mathrm{Li}$ and $\mathrm{Cao}$ [30] provided a risk decision analysis method by extending the TODIM method with interval numbers to solve multiattribute risk decision making problems in emergency response. Peng et al. [31] introduced a deviation-based method with q-rung orthopair fuzzy number (q-ROFN) to deal with EDM problems. In [32], a consistency-based EDM method was designed by extending the incomplete probabilistic linguistic term set in an incomplete probabilistic linguistic preference relation (InPLPR). In [33], a method combining probabilistic linguistic term sets (PLTSs) and the D-S evidence theory is applied to the EDM problem.

In addition, there are a few research studies that focused on EDM on the situation of the large group in recent years. Song and $\mathrm{Li}$ [34] proposed a consensus process of the large group EDM by using multigranular probabilistic fuzzy linguistic preference relations (MGPFLPRs) to represent subgroup's preferences information. Xu et al. [35] presented a framework for the LGEDM problem in a linguistic environment by considering decision makers' risk appetites. 
$\mathrm{Xu}$ et al. [36] found a LGEDM model for determining the severity of emergencies based on a decision paradigm obtained from the analysis of similar cases. $\mathrm{Xu}$ et al. [37] established a consensus model for the effective management of opinion differences and noncooperative behaviors in LGEDM environment. Cai et al. [16] suggested a multistage LGEDM model based on the preference information expressed as interval numbers and the similarity measures of decision makers. Aiming at the lower consensus and the urgency of LGEDM, Xu et al. [10] described a preference consensus model by taking into account the noncooperative behaviors and minority opinions of decision makers, and $\mathrm{Xu}$ et al. [38] proposed a dynamical consensus method based on exit-delegation mechanism considering consistency and consensus measures simultaneously.

The above literature review shows that various theories, such as interval numbers, 2-dimension uncertain linguistic variables, and probabilistic linguistic term sets, have been employed to deal with imprecise cognitive information in the EDM process. However, these theories are inefficient in expressing the reliability of decision makers' cognitive assessments. Moreover, no or little attention has been paid to the large group EDM problems under the context of linguistic $Z$-numbers. In addition, various MCDM methods have been proposed to deal with EDM problems. To the best of our knowledge, no previous studies have investigated EDM problems with the QUALIFLEX method yet. Therefore, in this paper, we fill the above gaps by developing a novel, integrated cognitive approach based on linguistic $Z$ numbers and an extended QUALIFLEX method to solve the EDM problem within the large group environment. The developed method in this paper can not only express the subjective cognitive evaluation information of decision makers more precisely but also support the rescue team in finding the optimal response to an emergency event effectively.

\section{Preliminaries}

3.1. Linguistic Z-Numbers. Combining Z-numbers [39] and linguistic variables [40], Wang et al. [6] introduced the concept of linguistic $Z$-numbers to address the reliability of information as a significant dimension in a decision making process.

Definition 1 (see [41]). An uncertain variable is a measurable function from an uncertainty space to the set of real numbers.

Definition 2 (see [6]). Suppose that $X$ is a finite universe of discourses. $S_{1}=\left(s_{0}, s_{1}, \ldots, s_{g-1}\right)$ and $S_{2}=\left(s_{0}^{\prime}, s_{1}^{\prime}, \ldots, s_{g^{\prime}-1}^{\prime}\right)$ are two finite and totally ordered discrete linguistic term sets, where $g$ and $g^{\prime}$ are the positive odd integers. Let $A_{\phi(x)} \in S_{1}$ and $B_{\varphi(x)} \in S_{2}$. A linguistic $Z$-number set $Z$ in $X$ is expressed as

$$
Z=\left\{\left(x, A_{\phi(x)}, B_{\varphi(x)}\right) \mid x \in X\right\}
$$

where $A_{\phi(x)}$ represents the fuzzy restriction on the domain of the uncertain variables, and $B_{\varphi(x)}$ is a measure of reliability of $A_{\phi(x)}$.

Normally, $S_{1}$ and $S_{2}$ represent different preference information and, hence, are not always consistent. For simplicity, $z=\left(A_{\phi(x)}, B_{\varphi(x)}\right)$ is called a linguistic $Z$-number, where $A_{\phi(x)} \in S_{1}$ and $B_{\varphi(x)} \in S_{2}$ are the two linguistic terms.

Definition 3 (see [6]). Let $z_{u}=\left(A_{\phi(u)}, B_{\varphi(u)}\right)$ and $z_{v}=\left(A_{\phi(v)}, B_{\varphi(v)}\right)$ be two linguistic $Z$-numbers and $f^{*}$ and $g^{*}$ be the possible functions of $F_{1}\left(\theta_{u}\right), F_{2}\left(\theta_{u}\right), F_{3}\left(\theta_{u}\right)$, and $F_{4}\left(\theta_{u}\right)$ where $F_{1}\left(\theta_{u}\right), F_{2}\left(\theta_{u}\right), F_{3}\left(\theta_{u}\right)$, and $F_{4}\left(\theta_{u}\right)$ are strictly monotonically increasing functions. Then, the operational laws of $Z$-numbers are defined as follows:

$$
\begin{aligned}
& \text { (1) } \operatorname{neg}\left(z_{u}\right)=\left(f^{*-1}\left(f^{*}\left(A_{l-1}\right)-f^{*}\left(A_{\phi(u)}\right)\right), g^{*-1}\left(g^{*}\right.\right. \\
&\left.\left(B_{k-1}\right)-g^{*}\left(B_{\varphi(u)}\right)\right) \\
& \text { (2) } z_{u} \oplus z_{v}=\left(f ^ { * - 1 } ( f ^ { * } ( A _ { \phi ( u ) } ) + f ^ { * } ( A _ { \phi ( v ) } ) ) g ^ { * - 1 } \left(\left(f^{*}\right.\right.\right. \\
&\left.\left(A_{\phi(u)}\right) \times g^{*}\left(B_{\varphi(u)}\right)+f^{*}\left(A_{\phi(v)}\right) \times g^{*}\left(B_{\varphi(v)}\right)\right) /\left(f^{*}\right. \\
&\left.\left.\left(A_{\phi(u)}\right)+f^{*}\left(A_{\phi(v)}\right)\right)\right) \\
& \text { (3) } z_{u} \otimes z_{v}=\left(f^{*-1}\left(f^{*}\left(A_{\phi(u)}\right) f^{*}\left(A_{\phi(v)}\right)\right), g^{*-1}\left(g^{*}\right.\right. \\
&\left.\left.\left(B_{\varphi(u)}\right) g^{*}\left(B_{\varphi(v)}\right)\right)\right) \\
& \text { (4) } \lambda z_{u}=\left(f^{*-1}\left(\lambda f^{*}\left(A_{\phi(u)}\right)\right), B_{\varphi(u)}\right), \text { for } \lambda \geq 0 \\
& \text { (5) }\left(z_{u}\right)^{\lambda}=\left(f^{*-1}\left(f^{*}\left(A_{\phi(u)}\right)\right), g^{*-1}\left(g^{*}\left(B_{\varphi(u)}\right)^{\lambda}\right)\right), \text { for } \\
& \quad \lambda \geq 0
\end{aligned}
$$

Definition 4 (see [6]). Suppose that $S=\left\{s_{0}, s_{1}, \ldots, s_{g-1}\right\}$ is a linguistic term set and $z_{u}=\left(A_{\phi(u)}, B_{\varphi(u)}\right)$ is a linguistic $Z$ number; then, the score function of $z_{u}$ is defined as

$$
S\left(z_{u}\right)=f^{*}\left(A_{\phi(u)}\right) \times g^{*}\left(B_{\varphi(u)}\right),
$$

and the accuracy function of $z_{u}$ is defined as

$$
A\left(z_{u}\right)=f^{*}\left(A_{\phi(u)}\right) \times\left(1-g^{*}\left(B_{\varphi(u)}\right)\right) .
$$

Definition 5 (see [6]). Let $z_{u}=\left(A_{\phi(u)}, B_{\varphi(u)}\right)$ and $z_{v}=$ $\left(A_{\phi(v)}, B_{\varphi(v)}\right)$ be two linguistic $Z$-numbers. Then, the comparison laws of $z_{u}$ and $z_{v}$ are defined as follows:

(1) When $A_{\phi(u)}>A_{\phi(v)}$ and $B_{\varphi(u)}>B_{\varphi(v)}$, then $z_{u}$ is strictly greater than $z_{v}$, expressed as $z_{u}>z_{v}$

(2) When $S\left(z_{u}\right) \geq S\left(z_{v}\right)$ and $A\left(z_{u}\right)>A\left(z_{v}\right)$, then $z_{u}$ is greater than $z_{v}$, expressed as $z_{u}>z_{v}$

(3) When $S\left(z_{u}\right)=S\left(z_{v}\right)$ and $A\left(z_{u}\right)=A\left(z_{v}\right)$, then $z_{u}$ equals $z_{v}$, expressed as $z_{u} \sim z_{v}$

(4) When $S\left(z_{u}\right) \leq S\left(z_{v}\right)$ and $A\left(z_{u}\right)<A\left(z_{v}\right)$, then $z_{u}$ is less than $z_{v}$, expressed as $z_{u}<z_{v}$

Definition 6 (see [6]). Let $z_{u}=\left(A_{\phi(u)}, B_{\varphi(u)}\right)$ and $z_{v}=$ $\left(A_{\phi(v)}, B_{\varphi(v)}\right)$ be two linguistic $Z$-numbers and $f^{*}$ and $g^{*}$ be two linguistic scale functions. Then, the distance between $z_{u}$ and $z_{v}$ is defined as 


$$
\begin{aligned}
d\left(z_{u}, z_{v}\right)= & \frac{1}{2}\left(\left|f^{*}\left(A_{\phi(u)}\right) \times g^{*}\left(B_{\varphi(u)}\right)-f^{*}\left(A_{\phi(v)}\right) \times g^{*}\left(B_{\varphi(v)}\right)\right|\right. \\
& \left.+\max \left\{\left|f^{*}\left(A_{\phi(u)}\right)-f^{*}\left(A_{\phi(v)}\right)\right|,\left|g^{*}\left(B_{\varphi(u)}\right)-g^{*}\left(B_{\varphi(v)}\right)\right|\right\}\right) .
\end{aligned}
$$

To aggregate the evaluations expressed by linguistic $Z$ numbers, the linguistic $Z$-number weighted averaging (LZNWA) operator is proposed.

Definition 7. Suppose $Z=\left\{z_{j} \mid j=1,2, \ldots, n\right\}$ is a collection of linguistic $Z$-numbers $z_{j}=\left(A_{\phi(j)}, B_{\varphi(j)}\right)$, and LZNWA: $Z^{n} \longrightarrow Z$, then the LZNWA operator is given by

$$
\operatorname{LZNWA}\left(z_{1}, z_{2}, \ldots, z_{n}\right)=\underset{j=1}{\oplus}\left(w_{j} z_{j}\right)
$$

where $\oplus$ is the same operator defined in Definition $3, w_{j}$ is the weight of $z_{j}$, satisfying $0 \leq w_{j} \leq 1$, for $j=1,2, \ldots, n$, and $\sum_{j=1}^{n} w_{j}=1$. If $w=(1 / n, 1 / n, \ldots, 1 / n)$, the LZNWA operator becomes the linguistic $Z$-number arithmetic mean (LZAM) operator.

3.2. QUALIFLEX Method. The QUALIFLEX method, proposed by Paelinck [25], is a useful outranking technique for solving multiple attribute decision making problems with exact values for the evaluations given by the decision makers [42, 43]. The QUALIFLEX method treats cardinal and ordinal information simultaneously in the process of decision making. In the QUALIFLEX method, pairwise comparisons of the alternatives with respect to each criterion under all possible alternative permutations are made, and the optimal alternative(s) can be determined by maximizing the value of the comprehensive concordance/ discordance index [44] among all possible alternative permutations. Let $E=\left\{E_{1}, E_{2}, \ldots, E_{m}\right\}$ be a set of alternatives, $C=\left\{C_{1}, C_{2}, \ldots, C_{n}\right\}$ be a set of criteria, and $W=$ $\left\{w_{1}, w_{2}, \ldots, w_{n}\right\}$ be the weights of the criteria satisfying $0 \leq w_{j} \leq 1$, for $j=1,2, \ldots, n$, and $\sum_{j=1}^{n} w_{j}=1$. There are $m$ ! permutations of the rankings of the alternatives. The decision process of the QUALIFLEX method is described step by step as follows $[25,45]$ :

Step 1: list all $m$ ! permutations of the rankings of the alternatives. Suppose $P_{\eta}$, as shown in equation (6), is the $\eta$ th permutation:

$$
P_{\eta}=\left(\ldots, E_{\alpha}, \ldots, E_{\beta}, \ldots\right), \quad \text { for } \eta=1,2, \ldots, m !,
$$

where $E_{\alpha}, E_{\beta} \in E$, and the ranking of $E_{\alpha}$ is higher or equal to that of $E_{\beta}$.

Step 2: determine the concordance/discordance index $I_{j}^{\eta}\left(E_{\alpha}, E_{\beta}\right)$ by using equation (7) for each pair of alternatives $\left(E_{\alpha}, E_{\beta}\right)$, and measure the similarity between $E_{\alpha}$ and $E_{\beta}$, in each permutation $P_{\eta}$ with respect to each criterion $j$ :

$$
I_{j}^{\eta}\left(E_{\alpha}, E_{\beta}\right)=\left\{\begin{array}{l}
E_{\alpha}>E_{\beta} \Leftrightarrow \text { concordance exists, } \\
E_{\alpha} \sim E_{\beta} \Leftrightarrow \text { ex aequo exists, } \\
E_{\alpha} \prec E_{\beta} \Leftrightarrow \text { discordance exists. }
\end{array}\right.
$$

Step 3: calculate the weighted concordance/discordance index $I^{\eta}\left(E_{\alpha}, E_{\beta}\right)$ by using equation (8) for each pair of alternatives $E_{\alpha}$ and $E_{\beta}$ in each permutation $P_{\eta}$ :

$$
I^{\eta}\left(E_{\alpha}, E_{\beta}\right)=\sum_{j=1}^{n} I_{j}^{\eta}\left(E_{\alpha}, E_{\beta}\right) w_{j}
$$

Step 4: determine the comprehensive concordance/ discordance index $I^{\eta}$ by using equation (9) for each permutation $P_{\eta}$ and determine the optimal alternative:

$$
I^{\eta}=\sum_{\alpha, \beta=1,2, \ldots, m} I^{\eta}\left(E_{\alpha}, E_{\beta}\right) .
$$

The best ranking of the alternatives is the one with the maximum value of the comprehensive concordance/discordance index $I^{*}=\max _{\eta=1,2, \ldots, m !}\left\{I^{\eta}\right\}$. The alternative ranked on the top of the corresponding ranking $P^{*}$ is the optimal alternative.

\section{The Proposed LGEDM Approach}

In this section, the linguistic Z-QUALIFLEX method, an extended QUALIFLEX approach with linguistic $Z$-numbers, is proposed to solve emergency decision making problems when a large group of decision makers is involved. The evaluations of the alternatives with respect to the criteria, represented by $Z$ numbers, are given by the large group of decision makers. The major steps of the proposed approach are shown in Figure 1. The decision makers are first divided into several subgroups according to the similarities of their evaluations. The evaluations represented by linguistic $Z$-numbers given by the decision makers in each subgroup are then aggregated into a group linguistic $Z$-number decision matrix using the LZAM operator. The best ranking of the alternatives is finally identified by using the QUALIFLEX method.

In the QUALIFLEX method, let $E=\left\{E_{1}, E_{2}, \ldots, E_{m}\right\}$ be a set of emergency solutions, i.e., alternatives, $C=\left\{C_{1}\right.$, $\left.C_{2}, \ldots, C_{n}\right\}$ be a set of criteria, $W=\left\{w_{1}, w_{2}, \ldots, w_{n}\right\}$ be the weights of the criteria satisfying $0 \leq w_{j} \leq 1$, for $j=1,2, \ldots, n$, and $\sum_{j=1}^{n} w_{j}=1$, and $\mathrm{DM}=\left\{D_{1}, D_{2}, \ldots, D_{l}\right\}$ with $l \geq 20$ be a set of decision makers in an LGEDM problem. Each decision maker $D_{k}$ gives his/her judgment of $E_{i}$ with respect to $C_{j}$ using linguistic $Z$-numbers, represented by $z_{i j}^{k}$. The linguistic $Z$-number evaluation matrices $Z_{k}=\left[z_{i j}^{k}\right]_{m \times n}$, with $i=1,2$, $\ldots, m$ and $j=1,2, \ldots, n$ for $k=1,2, \ldots, l$, are then obtained. Each element $z_{i j}^{k}$ of $Z_{k}$ is given by $z_{i j}^{k}=\left(A_{\phi(i j)}^{k}\right.$, $\left.B_{\varphi(i j)}^{k}\right)$, where $A_{\phi(i j)}^{k}$ is the linguistic evaluation of alternative $E_{i}$ with respect to criterion $C_{j}$ given by the decision maker 


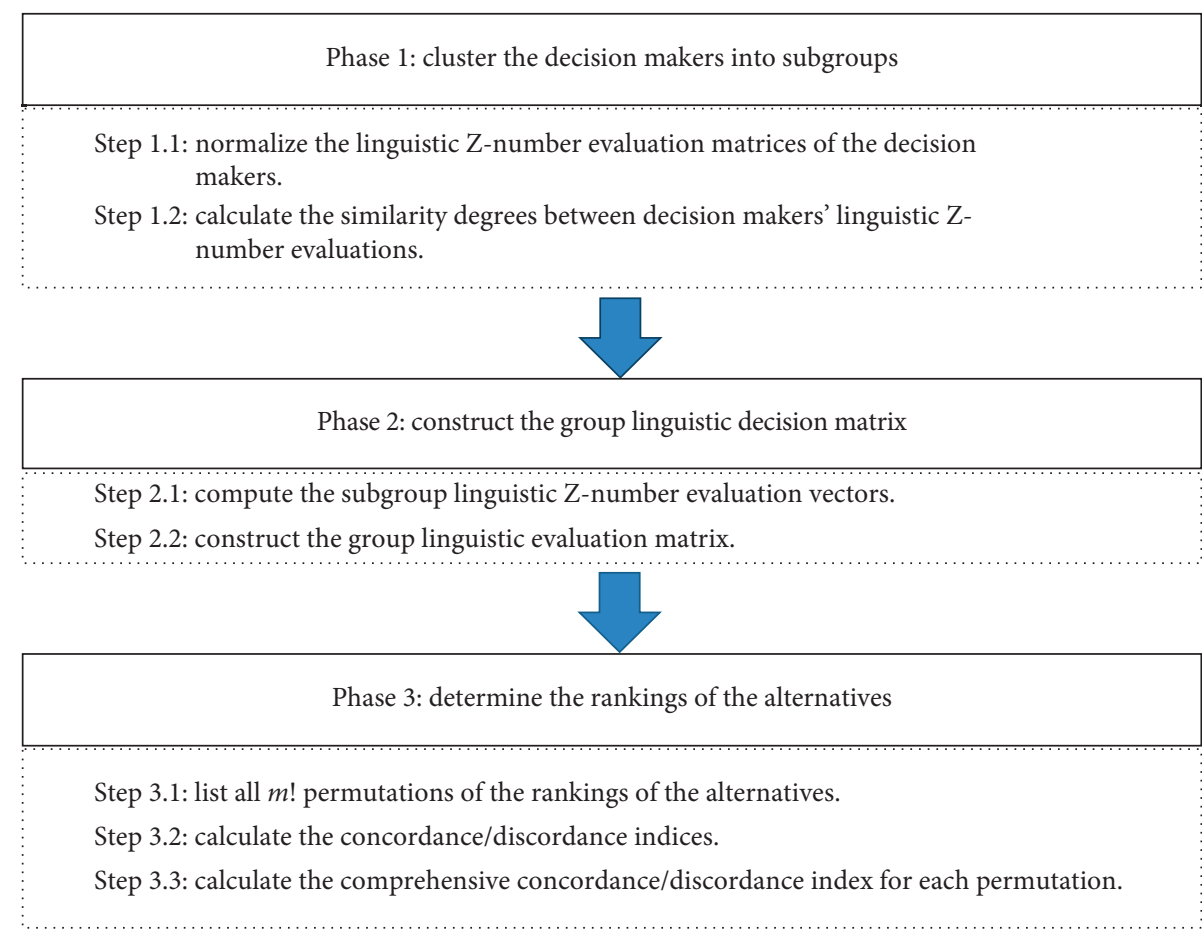

FIGURE 1: Flowchart of the proposed linguistic Z-QUALIFLEX method.

$D_{k}$ using the linguistic term set $S_{1}=\left(s_{0}, s_{1}, \ldots, s_{g-1}\right)$, and $B_{\varphi(i j)}^{k}$ is a measure of reliability of $A_{\phi(i j)}^{k}$ using the linguistic term set $S_{2}=\left(s_{0}^{\prime}, s_{1}^{\prime}, \ldots, s_{g^{\prime}-1}^{\prime}\right)$. The evaluations of decision makers are assumed to be independent of each other. The linguistic Z-QUALIFLEX method used to solve LGEDM problems is presented step by step in three phases in the following.

Phase 1: cluster the decision makers into subgroups

Step 1.1: normalize the linguistic $Z$-number evaluation matrix $Z_{k}=\left[z_{i j}^{k}\right]_{m \times n}$ by using

$$
\bar{z}_{i j}^{k}= \begin{cases}z_{i j}^{k}, & \text { for benefit criteria, } \\ \operatorname{neg}\left(z_{i j}^{k}\right), & \text { for cost criteria. }\end{cases}
$$

The normalized linguistic $Z$-number evaluation matrix is represented by $\bar{Z}_{k}=\left[\bar{z}_{i j}^{k}\right]_{m \times n}$.

Step 1.2: calculate the similarity degrees between decision makers' linguistic $Z$-number evaluations. Let $V_{k}^{(i)}=\left(\bar{z}_{i 1}^{k}, \bar{z}_{i 2}^{k}, \ldots, \bar{z}_{i n}^{k}\right)$ denote the normalized linguistic evaluation vector for alternative $E_{i}$, for $i=1,2, \ldots, m$, by decision maker $k$, for $k=1,2, \ldots, l$. The similarity degree $S\left(V_{\mu}^{(i)}, V_{\nu}^{(i)}\right)$ between $V_{\mu}^{(i)}$ and $V_{\nu}^{(i)}$ is given by

$$
S\left(V_{\mu}^{(i)}, V_{\nu}^{(i)}\right)=\frac{1}{1+\sum_{j=1}^{n} d\left(\bar{z}_{i j}^{\mu}, \bar{z}_{i j}^{v}\right)} .
$$

Step 1.3: divide decision makers into subgroups for each alternative. Suppose $\lambda^{(i)}$ is the clustering threshold of alternative $E_{i}$ obtained by using

$$
\lambda^{(i)}=\operatorname{SMin}+\frac{2}{3}(\operatorname{SMax}-\operatorname{SMin})
$$

where

$$
\begin{aligned}
& S \operatorname{Max}=\max \left\{S\left(V_{\mu}^{(i)}, V_{\nu}^{(i)}\right) \mid V_{\mu}^{(i)}, V_{\nu}^{(i)} \in \Omega^{(i)}, V_{\mu}^{(i)} \neq V_{\nu}^{(i)}\right\}, \\
& S \operatorname{Min}=\min \left\{S\left(V_{\mu}^{(i)}, V_{\nu}^{(i)}\right) \mid V_{\mu}^{(i)}, V_{\nu}^{(i)} \in \Omega^{(i)}, V_{\mu}^{(i)} \neq V_{\nu}^{(i)}\right\},
\end{aligned}
$$

and $\Omega^{(i)}$ are the evaluation vectors of all decision makers for alternative $E_{i}$.

For alternative $E_{i}$, if $S\left(V_{\mu}^{(i)}, V_{\nu}^{(i)}\right) \geq \lambda^{(i)}$, then $V_{\mu}^{(i)}$ and $V_{\nu}^{(i)}$ are put into the same subgroup. Let ${ }_{H}^{(i)}$ denote the number of subgroups after clustering for alternative $E_{i}$, $\Lambda_{h}^{(i)}$ be the decision makers in subgroup $h, \Omega_{h}^{(i)}$ be the corresponding assessment vectors of $\Lambda_{h}^{(i)}$, and $k_{h}^{(i)}$ be the number of decision makers in $\Lambda_{h}^{(i)}$. The requirement $k_{h}^{(i)} \geq 2$ is needed. If there is a $\Lambda_{\xi}^{(i)}$, for any $\xi=$ $1,2, \ldots, H^{(i)}$, such that $k_{\xi}^{(i)}=1, \Lambda_{\xi}^{(i)}$ needs to be modified. The method proposed by Cai et al. [16] is used for modification.

Phase 2: construct the group linguistic decision matrix

Step 2.1: aggregate the linguistic $Z$-number evaluation vectors of the decision makers in each subgroup into a subgroup linguistic $Z$-number evaluation vector by the LZAM operator. For each alternative $E_{i}$, the linguistic $Z$-number evaluation vectors $\Omega_{h(i)}^{(i)}$, for $1 \leq h \leq_{H}^{(i)}$, of $k_{h}^{(i)}$ decision makers in subgroup $\Lambda_{h}^{(i)}$ are aggregated into the subgroup linguistic evaluation vector $\widetilde{V}_{h}^{(i)}$ by using the LZAM operator: 


$$
\widetilde{V}_{h}^{(i)}=\operatorname{LZAM}\left(\bar{V}_{1}^{(i)}, \bar{V}_{2}^{(i)}, \ldots, \bar{V}_{k_{h}^{(i)}}^{(i)}\right)={\underset{r=1}{k_{h}^{(i)}}}_{r=1}\left(\frac{1}{k_{h}^{(i)}} \bar{z}_{i j r}^{(i)}\right),
$$

where $\left(1 / k_{h}^{(i)}\right)$ is the weight of $\bar{z}_{i j r}^{(i)}$.

Step 2.2: construct the group linguistic evaluation matrix $\widehat{Z}=\left[\widehat{z}_{i j}\right]_{m \times n}$. Using the subgroup linguistic evaluation vectors $\widetilde{V}_{h}^{(i)}$, for $i=1,2, \ldots, m$, the group linguistic evaluation vector can be obtained by using the LZAM operator:

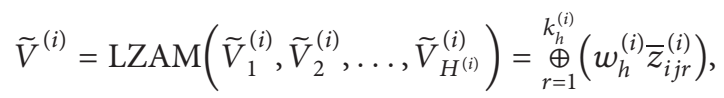

where $w_{h}^{(i)}=\left(k_{h}^{(i)} / l\right)$. The $m$ group linguistic evaluation vectors $V^{(i)}$, for $i=1,2, \ldots, m$, form the group linguistic evaluation matrix $\widehat{Z}=\left[\widehat{z}_{i j}\right]_{m \times n}$.

Phase 3: rank the alternatives by using the QUALIFLEX method

Step 3.1: list all $m$ ! permutations of the rankings of the alternatives:

$$
P_{\eta}=\left(\ldots, E_{\alpha}, \ldots, E_{\beta}, \ldots\right), \quad \text { for } \eta=1,2, \ldots, m !,
$$

where $P_{\eta}$ is the $\eta$ th permutation, $E_{\alpha}, E_{\beta} \in E$, and $E_{\alpha}$ is ranked higher or equal to $E_{\beta}$ in the permutation.

Step 3.2: calculate the concordance/discordance index $I_{j}^{\eta}\left(E_{\alpha}, E_{\beta}\right)$ of each pair of alternatives $\left(E_{\alpha}, E_{\beta}\right)$ in each permutation $P_{\eta}$ by considering every criterion using

$$
I_{j}^{\eta}\left(E_{\alpha}, E_{\beta}\right)=\left\{\begin{array}{l}
d\left(z_{\alpha j}, z_{0}\right)-d\left(z_{\beta j}, z_{0}\right)>0 \Leftrightarrow \text { concordance exists, } \\
d\left(z_{\alpha j}, z_{0}\right)-d\left(z_{\beta j}, z_{0}\right)=0 \Leftrightarrow \text { ex aequo exists, } \\
d\left(z_{\alpha j}, z_{0}\right)-d\left(z_{\beta j}, z_{0}\right)<0 \Leftrightarrow \text { discordance exists. }
\end{array}\right.
$$

Step 3.3: calculate the weighted concordance/discordance index $I^{\eta}\left(E_{\alpha}, E_{\beta}\right)$ of each permutation $P_{\eta}$ by using

$$
I^{\eta}\left(E_{\alpha}, E_{\beta}\right)=\sum_{j=1}^{n} I_{j}^{\eta}\left(E_{\alpha}, E_{\beta}\right) w_{j} .
$$

Step 3.4: determine the comprehensive concordance/ discordance index $I^{\eta}$ by using

$$
I^{\eta}=\sum_{\alpha, \beta=1,2, \ldots, m} \sum_{j=1}^{n} I_{j}^{\eta}\left(E_{\alpha}, E_{\beta}\right) w_{j}
$$

The best ranking of the alternatives is $P^{*}$ with the maximum value of the comprehensive concordance/discordance index $I^{*}$, i.e., $I^{*}=\max _{\eta=1,2, \ldots, m !}\left\{I^{\eta}\right\}$. The alternative ranked on the top of $P^{*}$ is the optimal solution for the LGEDM problem.

\section{A Numerical Example}

In China, from June to July, there are often persistent rainstorms in the middle and lower reaches of the Yangtze River, which are of long duration, wide area, and heavy rainfall. July to August is the main rainstorm season in northern provinces, and the rainstorm intensity is very high. These torrential rains often lead to floods, which may cause river levee breach incidents, resulting in great harm to people and public property.

In order to reduce the huge damage to people and public property caused by these accidents and provide scientific support for the emergency management of such emergencies, in this section, an LGEDM problem of a river levee breach incident is used as the illustrative example to demonstrate the effectiveness and practicability of the proposed linguistic Z-QUALIFLEX method.

5.1. Decision Process and Results. In the morning of June 21, 2017, a severe thunderstorm watch with red color warning of possible heavy rainfall in the next three days was issued by the meteorological department in area $C$ in Hubei province of China. According to weather report, the probabilities of moderate, heavy, and violent rainfalls are $0.30,0.50$, and 0.20 , respectively. A riverbank breach will cause a wide range flooding disaster and a large number of casualties, seriously affecting the lives of people in the surrounding area. 20 experts from the emergency management department, the meteorological department, the water conservancy department, and the transportation department were invited to choose the best emergency solution within a short period of time. There are three emergency solutions $E=\left\{E_{1}, E_{2}, E_{3}\right\}$ for the decision makers to choose from. $E_{1}$ : strengthen the riverbank, keep close monitoring, and expand the emergency force of inspection and rescue. $E_{2}$ : strengthen the riverbank, keep close monitoring, send the villager rescue team to the site, and transfer the villagers in low-lying areas to safe areas. $E_{3}$ : strengthen the riverbank, keep close monitoring, and send the villager rescue team and medical team to the site, allocate a large quantity of relief supplies, and transfer the nearby villagers to safe areas. Four criteria $C=\left\{C_{1}, C_{2}, C_{3}, C_{4}\right\}$ are considered for each emergency solution: $C_{1}$-number of victims, $C_{2}$-public satisfaction, $C_{3}$-property loss, and $C_{4}$-rescue cost. The weights of these criteria are supposed as $W=(0.30,0.25,0.30$, and 0.15). Suppose that all decision makers $\mathrm{DM}=\left\{D_{1}, D_{2}, \ldots, D_{20}\right\}$ have the same weights and are required to give their 
linguistic $Z$-number evaluations for the solutions over each criterion. Note that $C_{2}$ is a benefit, i.e., maximization, criterion, while $C_{1}, C_{3}$, and $C_{4}$ are cost, i.e., minimization, criteria.
The evaluations given by the 20 decision makers on three solutions by considering the four criteria are represented by linguistic $Z$-numbers. The linguistic term set $S_{1}$ for $A_{\phi(i j)}^{k}$ used by the decision makers is

$$
\begin{aligned}
S_{1}=\left\{s_{0}\right. & =\text { very low influence, } s_{1}=\text { low influence, } s_{2}=\text { slightly low influence, } \\
s_{3} & =\text { moderate influence, } s_{4}=\text { slightly high influence, } s_{5}=\text { high influence, } \\
s_{6} & =\text { very high influence }\},
\end{aligned}
$$

and the linguistic term set $S_{2}$ for $B_{\varphi(i j)}^{k}$ is

$$
\begin{gathered}
S_{2}=\left\{s_{0,}=\text { very unreliable, } s_{1,}=\text { unreliable, } s_{2,}=\right.\text { somewhat reliable, } \\
\left.s_{3,}=\text { reliable, } s_{4,}=\text { very reliable }\right\} .
\end{gathered}
$$

The decision process and the results of the proposed linguistic Z-QUALIFLEX method of selecting the best solution for this example LGEDM problem are summarized as follows:

Phase 1: divide the decision makers into subgroups

The linguistic $Z$-number evaluation matrices are obtained first from all the decision makers. By using equation (10), the linguistic $Z$-number evaluation matrices of the decision makers are normalized. As a result, a normalized linguistic evaluation matrix composed of the evaluation vectors of 20 decision makers is constructed for each alternative. Due to space limitation, only $V_{k}^{(1)}=\left(\bar{z}_{k 1}^{(1)}, \bar{z}_{k 2}^{(1)}, \ldots, \bar{z}_{k 4}^{(1)}\right)$, for $k=$ $1,2, \ldots, 20$, i.e., the normalized linguistic evaluation vectors by the 20 decision makers for alternative $E_{1}$, are listed, which are shown in Table 1.

The similarity degrees between the decision makers' linguistic evaluation vectors of each alternative are then calculated by using equation (11). The result for alternative $E_{1}$ is shown in Table 2. By using equation (12) and the steps of modification, the decision makers for each alternative are divided into several subgroups, and the clustering results of $E_{1}$ are shown in Table 3.

Phase 2: construct the group linguistic decision matrix For each alternative $E_{i}$, for $i=1,2,3$, the linguistic $Z$ number evaluation vectors in $\Omega_{h}^{(i)}$, for $1 \leq h \leq_{H}^{(i)}$, of the $k_{h}^{(i)}$ decision makers in subgroup $\Lambda_{h}^{(i)}$ are aggregated into the subgroup linguistic evaluation vector $\widetilde{V}_{h}^{(i)}$ by using equation (14). By aggregating the subgroup linguistic evaluation vectors of all alternatives based on equation (15), the group linguistic evaluation matrix $\widehat{Z}=\left[\widehat{z}_{i j}\right]_{3 \times 4}$ is obtained as shown in Table 4.

Phase 3: rank the alternatives using the QUALIFLEX method
By equation (16), there are $6(=3$ !) permutations of the rankings for the three candidate emergency solutions, i.e.,

$$
\begin{aligned}
& P_{1}=\left(E_{1}, E_{2}, E_{3}\right), \\
& P_{2}=\left(E_{1}, E_{3}, E_{2}\right), \\
& P_{3}=\left(E_{2}, E_{1}, E_{3}\right), \\
& P_{4}=\left(E_{2}, E_{3}, E_{1}\right), \\
& P_{5}=\left(E_{3}, E_{1}, E_{2}\right), \\
& P_{6}=\left(E_{3}, E_{2}, E_{1}\right) .
\end{aligned}
$$

By using equation (17), the concordance/discordance index $I_{j}^{\eta}\left(E_{\alpha}, E_{\beta}\right)$ of each pair of solutions $\left(E_{\alpha}, E_{\beta}\right)$, for $\alpha, \beta=1,2,3$, in each permutation $P_{\eta}$, for $\eta=1,2, \ldots, 6$, is calculated by considering each criterion. Because of space limitation, only the concordance/discordance indices of permutation $P_{1}$ are presented in Table 5. The weighted concordance/discordance indices $I^{\eta}\left(E_{\alpha}, E_{\beta}\right)$ of each permutation $P_{\eta}$ are obtained by using equation (18), and the results of permutation $P_{1}$ are listed in Table 6 . Finally, the comprehensive concordance/discordance indices $I^{\eta}$ are calculated by using equation (19), and the results are $I^{1}=6.268, I^{2}=6.63, I^{3}=-0.362, I^{4}=6.624, I^{5}=0.87$, and $I^{6}=-5.754$, respectively. Hence, the best ranking of the alternatives is $E_{1}>E_{3}>E_{2}$. Therefore, $E_{1}$ is the best emergency solution.

5.2. Comparative Analysis. To demonstrate the effectiveness and practicability of the proposed linguistic Z-QUALIFLEX method, a comparative analysis is conducted. Four typical large group decision making methods at present including the interval type-2 fuzzy TOPSIS method [12], the preference conflict method [16], the large group EDM method considering experts' hesitation preference [46], and the large group decision making method based on expert's consensus [47] are used for comparison. The ranking results of the 
TABLE 1: The normalized linguistic evaluation matrix of the 20 decision makers for alternative $E_{1}$.

\begin{tabular}{|c|c|c|c|c|}
\hline $\mathrm{DM}$ & $C_{1}$ & $C_{2}$ & $C_{3}$ & $C_{4}$ \\
\hline$\overline{D_{1}}$ & $\left(A_{4}, B_{3}\right)$ & $\left(A_{3}, B_{2}\right)$ & $\left(A_{4}, B_{2}\right)$ & $\left(A_{1}, B_{4}\right)$ \\
\hline$D_{2}$ & $\left(A_{5}, B_{2}\right)$ & $\left(A_{4}, B_{2}\right)$ & $\left(A_{4}, B_{2}\right)$ & $\left(A_{4}, B_{2}\right)$ \\
\hline$D_{3}$ & $\left(A_{3}, B_{2}\right)$ & $\left(A_{4}, B_{3}\right)$ & $\left(A_{3}, B_{1}\right)$ & $\left(A_{3}, B_{2}\right)$ \\
\hline$D_{4}$ & $\left(A_{6}, B_{2}\right)$ & $\left(A_{2}, B_{3}\right)$ & $\left(A_{4}, B_{4}\right)$ & $\left(A_{1}, B_{4}\right)$ \\
\hline$D_{5}$ & $\left(A_{6}, B_{2}\right)$ & $\left(A_{1}, B_{3}\right)$ & $\left(A_{5}, B_{3}\right)$ & $\left(A_{3}, B_{2}\right)$ \\
\hline$D_{6}$ & $\left(A_{5}, B_{4}\right)$ & $\left(A_{2}, B_{3}\right)$ & $\left(A_{2}, B_{2}\right)$ & $\left(A_{2}, B_{4}\right)$ \\
\hline$D_{7}$ & $\left(A_{1}, B_{2}\right)$ & $\left(A_{1}, B_{1}\right)$ & $\left(A_{3}, B_{1}\right)$ & $\left(A_{2}, B_{1}\right)$ \\
\hline$D_{8}$ & $\left(A_{3}, B_{1}\right)$ & $\left(A_{3}, B_{1}\right)$ & $\left(A_{3}, B_{1}\right)$ & $\left(A_{3}, B_{1}\right)$ \\
\hline$D_{9}$ & $\left(A_{4}, B_{1}\right)$ & $\left(A_{3}, B_{1}\right)$ & $\left(A_{6}, B_{4}\right)$ & $\left(A_{1}, B_{1}\right)$ \\
\hline$D_{10}$ & $\left(A_{6}, B_{4}\right)$ & $\left(A_{2}, B_{2}\right)$ & $\left(A_{3}, B_{0}\right)$ & $\left(A_{2}, B_{4}\right)$ \\
\hline$D_{11}$ & $\left(A_{3}, B_{0}\right)$ & $\left(A_{3}, B_{0}\right)$ & $\left(A_{4}, B_{3}\right)$ & $\left(A_{3}, B_{1}\right)$ \\
\hline$D_{12}$ & $\left(A_{6}, B_{4}\right)$ & $\left(A_{1}, B_{3}\right)$ & $\left(A_{2}, B_{1}\right)$ & $\left(A_{2}, B_{2}\right)$ \\
\hline$D_{13}$ & $\left(A_{3}, B_{1}\right)$ & $\left(A_{3}, B_{1}\right)$ & $\left(A_{6}, B_{3}\right)$ & $\left(A_{3}, B_{1}\right)$ \\
\hline$D_{14}$ & $\left(A_{6}, B_{4}\right)$ & $\left(A_{6}, B_{3}\right)$ & $\left(A_{3}, B_{2}\right)$ & $\left(A_{0}, B_{4}\right)$ \\
\hline$D_{15}$ & $\left(A_{1}, B_{2}\right)$ & $\left(A_{1}, B_{2}\right)$ & $\left(A_{3}, B_{0}\right)$ & $\left(A_{1}, B_{2}\right)$ \\
\hline$D_{16}$ & $\left(A_{5}, B_{2}\right)$ & $\left(A_{3}, B_{0}\right)$ & $\left(A_{4}, B_{1}\right)$ & $\left(A_{3}, B_{0}\right)$ \\
\hline$D_{17}$ & $\left(A_{5}, B_{1}\right)$ & $\left(A_{4}, B_{1}\right)$ & $\left(A_{6}, B_{3}\right)$ & $\left(A_{3}, B_{1}\right)$ \\
\hline$D_{18}$ & $\left(A_{6}, B_{3}\right)$ & $\left(A_{1}, B_{3}\right)$ & $\left(A_{5}, B_{3}\right)$ & $\left(A_{3}, B_{3}\right)$ \\
\hline$D_{19}$ & $\left(A_{6}, B_{4}\right)$ & $\left(A_{1}, B_{1}\right)$ & $\left(A_{4}, B_{2}\right)$ & $\left(A_{2}, B_{1}\right)$ \\
\hline$D_{20}$ & $\left(A_{3}, B_{2}\right)$ & $\left(A_{3}, B_{2}\right)$ & $\left(A_{4}, B_{2}\right)$ & $\left(A_{4}, B_{3}\right)$ \\
\hline
\end{tabular}

three emergency solutions obtained by these three methods are shown in Figure 2.

From Figure 2, it can be seen that the rankings of the emergency solutions obtained by the proposed method are the same as those of the interval type-2 fuzzy TOPSIS method and the large group EDM method considering experts' hesitation preference, but the rankings are different from those of the preference conflict method and the large group decision making method based on experts' consensus. Compared with the interval type-2 fuzzy TOPSIS method and the large group EDM method considering experts' hesitation preference, the calculation process is simpler by the proposed method.

The ranking result obtained with the proposed method is more practical. The reasons are as follows: in this example, first, the best solution $E_{1}$ selected by the proposed method not only ensures public safety but also saves cost. The probability of a violent rainfall causing collapse of the riverbank in area $C$ is 0.20 , which means that the probability of collapse of the riverbank is small. The solution $E_{3}$ involves sending the villager rescue team, the medical and the health team to the site, allocating a large quantity of relief supplies, and transferring the nearby villagers, which requires large quantities of manpower, material, and financial resources. If $E_{3}$ is taken as the emergency solution under the situation of a relatively small probability of collapse of the riverbank, it may not only cause wastes of the manpower, material, and financial resources but also lead to the discontent of the transferred villagers, which can minimize casualties but costs the most. $E_{2}$ cannot minimize casualties or improve personnel satisfaction, and it needs more material consumption compared with $E_{1}$. Therefore, it is more reasonable for the emergency management office to inform all kinds of emergency organizations and villagers in advance to prepare for the emergency and the transfer, and to strengthen the riverbank, keep close monitoring, and expand the emergency force of inspection and rescue at the same time just as what the alternative $E_{1}$ will do. Once a breach of the riverbank happens, the villagers will be transferred immediately, and less manpower, material, and financial resources are needed. Besides, in reality, a river levee breach occurred in Matou village in the afternoon of June 23, 2017. The whole village was in danger of flooding, and the lives and property of 2,600 people in the village were threatened. The emergency solution $E_{1}$ is also in agreement with the emergency measure taken in reality by the emergency management office to the river levee breach incident occurred in area $C$.

Comparing with the existing LGEDM methods, the linguistic Z-QUALIFLEX method has the following advantages: (1) the linguistic $Z$-numbers can describe the inherent thoughts of the decision makers more precisely and practically, ensuring the accuracy of the final results. (2) The similarity degrees and the LZAM operator are used for dividing the large group of decision makers into subgroups and for aggregating subgroups, which not only takes the conflicting nature of large group decision preferences into account but also considers the subgroup opinions under different preferences. (3) The QUALIFLEX method based on preference relation similarities is used to rank the emergency solutions. This method can handle cardinal and ordinal information in the process of decision making at the same time and can well express the complexity of the LGEDM problem. (4) Many existing large group decision making methods need to constantly recalculate expert weights or other parameters to classify expert groups, and the initial subgroups of experts or the value of some thresholds are mostly set artificially, which not only increases the calculation complexity but also may cause the decision results change due to different parameter values. The proposed method adopts an automatic threshold determination method to classify decision makers. The calculation is 


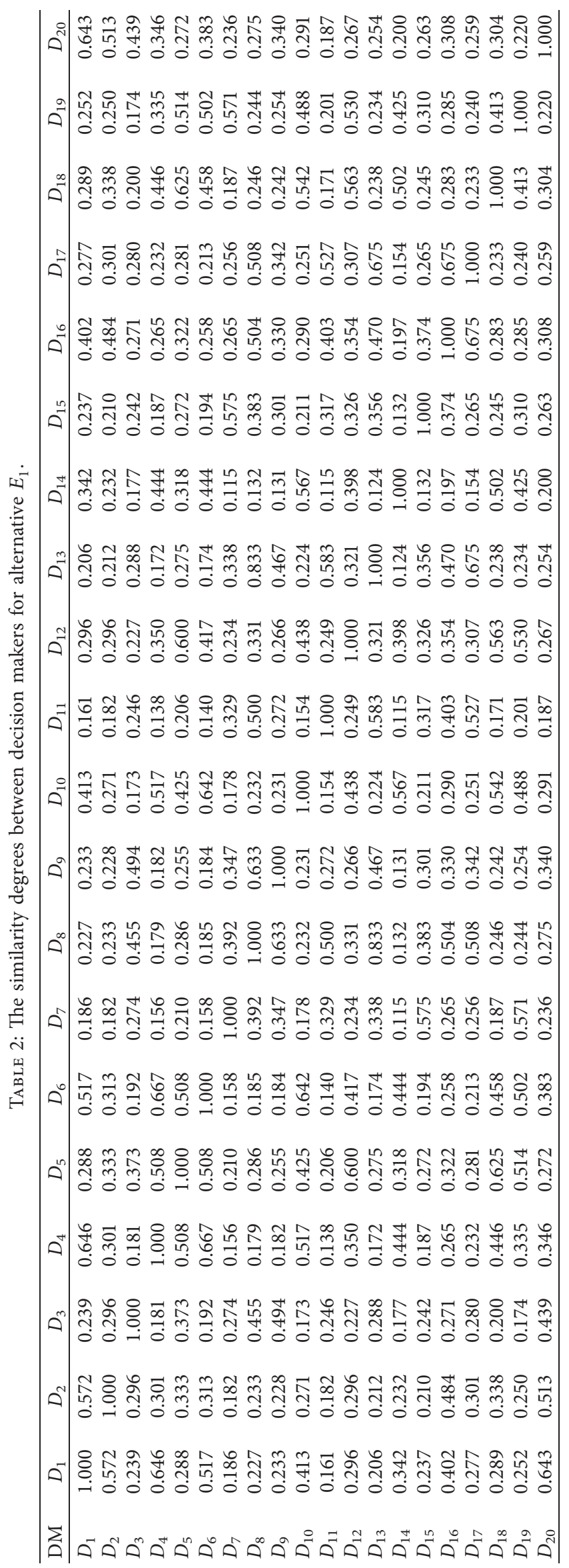


TABLE 3: The subgroups for each of the alternatives.

\begin{tabular}{lc}
\hline Alternatives & Subgroups \\
\hline$E_{1}$ & $\left\{D_{8}, D_{13}\right\} ;\left\{D_{1}, D_{4}, D_{5}, D_{6}, D_{10}, D_{18}, D_{20}\right\} ;\left\{D_{16}, D_{17}\right\} ;\left\{D_{3}, D_{7}, D_{9}, D_{12}, D_{14}, D_{19}\right\} ;\left\{D_{2}, D_{11}, D_{15}\right\}$. \\
$E_{2}$ & $\left\{D_{1}, D_{2}, D_{4}, D_{6}, D_{7}, D_{11}, D_{13}, D_{14}, D_{16}, D_{17}, D_{18}, D_{19}\right\} ;\left\{D_{3}, D_{8}, D_{15}, D_{20}\right\} ;\left\{D_{5}, D_{9}, D_{10}, D_{12}\right\}$. \\
$E_{3}$ & $\left\{D_{1}, D_{2}, D_{4}, D_{5}, D_{6}, D_{10}, D_{12}, D_{13}, D_{14}, D_{15}, D_{16}, D_{17}, D_{18}, D_{19}, D_{20}\right\} ;\left\{D_{3}, D_{8}\right\} ;\left\{D_{7}, D_{9}, D_{11}\right\}$. \\
\hline
\end{tabular}

TABLE 4: The group linguistic evaluation matrix.

\begin{tabular}{lcccc}
\hline Alternatives & $C_{1}$ & $C_{2}$ & $C_{3}$ & $C_{4}$ \\
\hline$E_{1}$ & $\left(A_{3.400}, B_{3.997}\right)$ & $\left(A_{5.119}, B_{2.145}\right)$ & $\left(A_{4.633}, B_{4.337}\right)$ & $\left(A_{5.346}, B_{4.221}\right)$ \\
$E_{2}$ & $\left(A_{1.118}, B_{0.889}\right)$ & $\left(A_{2.264}, B_{1.521}\right)$ & $\left(A_{1.056}, B_{0.708}\right)$ & $\left(A_{1.014}, B_{0.826}\right)$ \\
$E_{3}$ & $\left(A_{0.991}, B_{0.880}\right)$ & $\left(A_{4.168}, B_{2.636}\right)$ & $\left(A_{1.093}, B_{1.084}\right)$ & $\left(A_{0.920}, B_{0.622}\right)$ \\
\hline
\end{tabular}

TABle 5: The concordance/discordance indices for $P_{1}$.

\begin{tabular}{|c|c|c|c|c|c|c|}
\hline$P_{1}$ & $I_{j}^{1}\left(E_{1}, E_{2}\right)$ & $I_{j}^{1}\left(E_{1}, E_{3}\right)$ & $I_{j}^{1}\left(E_{2}, E_{1}\right)$ & $I_{j}^{1}\left(E_{2}, E_{3}\right)$ & $I_{j}^{1}\left(E_{3}, E_{1}\right)$ & $I_{j}^{1}\left(E_{3}, E_{2}\right)$ \\
\hline$\overline{C_{1}}$ & 2.898 & 3.005 & -2.898 & 0.127 & -3.005 & 0.127 \\
\hline$C_{2}$ & -2.855 & -3.005 & 2.855 & -1.904 & 3.005 & 1.904 \\
\hline$C_{3}$ & 3.608 & 3.570 & -3.608 & -0.038 & -3.570 & 0.038 \\
\hline$C_{4}$ & 4.350 & 4.444 & -4.350 & 0.094 & -4.444 & -0.094 \\
\hline
\end{tabular}

TABle 6: The weighted concordance/discordance indices for $P_{1}$.

\begin{tabular}{ccccccc}
\hline & $I_{j}^{1}\left(E_{1}, E_{2}\right)$ & $I_{j}^{1}\left(E_{1}, E_{3}\right)$ & $I_{j}^{1}\left(E_{2}, E_{1}\right)$ & $I_{j}^{1}\left(E_{2}, E_{3}\right)$ & $I_{j}^{1}\left(E_{3}, E_{1}\right)$ & $I_{j}^{1}\left(E_{3}, E_{2}\right)$ \\
\hline$P_{1}$ & 3.312 & 3.391 & -3.312 & -0.435 & -3.391 & 0.435 \\
$P_{2}$ & 3.318 & 2.877 & -3.318 & -0.435 & -2.877 & -2.877 \\
$P_{3}$ & 3.312 & 2.877 & -3.312 & 0.073 & -2.877 & -0.073 \\
$P_{4}$ & 3.312 & 2.877 & -3.312 & -0.435 & -2.877 & 0.435 \\
$P_{5}$ & 3.312 & 2.877 & -3.312 & -0.435 & -2.877 \\
$P_{6}$ & 3.312 & 2.877 & -3.312 & -0.435 & 0.435 \\
\hline
\end{tabular}

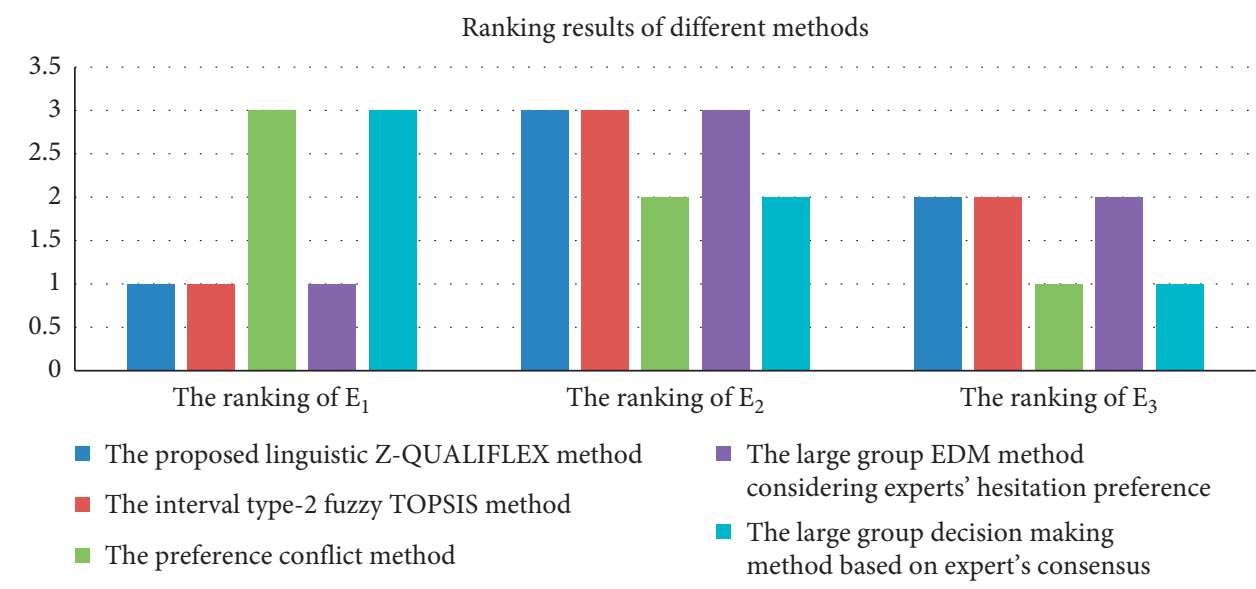

FIGURE 2: Ranking results of different methods.

relatively simple and helps to reduce and eliminate the decision making risk caused by subjectivity.

\section{Conclusions}

This study proposed an extended QUALIFLEX method using linguistic $Z$-numbers, called the linguistic Z-QUALIFLEX method, to deal with the LGEDM problem. Linguistic $Z$ numbers are first used to express the evaluations of the decision makers, which can more precisely express the inherent opinions of the decision makers. The similarity degrees between the decision makers' linguistic evaluation vectors of each alternative are then calculated and used to divide the large group of decision makers into several subgroups. The 
evaluations of the decision makers in each subgroup are aggregated, and the group linguistic decision matrix is constructed by the LZAM operator. The QUALIFLEX method is finally used to rank the emergency solutions. To demonstrate the effectiveness and practicability of the proposed linguistic $Z$-QUALIFLEX method, a real-life example of a river levee breach incident is presented, and the obtained ranking result is compared with those of the existing LGEDM methods.

There are some improvements to be made in future studies. (1) The proposed method should be extended to support LGEDM problems by considering more complex influencing factors. (2) The complex psychological behaviors of the decision makers in the decision making process under uncertain or emergency circumstances should be considered. (3) More linguistic expression techniques should be investigated for reducing the subjectivity in the decision making process. (4) More reasonable and effective approaches for dividing a large group of decision makers into subgroups need to be developed.

\section{Data Availability}

The data used to support the findings of this study are available from the corresponding author upon request.

\section{Conflicts of Interest}

The authors declare that there are no conflicts of interest regarding the publication of this paper.

\section{Acknowledgments}

This work was partially supported by the National Natural Science Foundation of China (No. 71502098), the Soft Science Research Support Project of Shanghai Science and Technology Development Fund (No. 19692109000), and the Training program of School of management of Shanghai University (2020-SDGY-KZ-003).

\section{References}

[1] L. Wang, Y.-M. Wang, and L. Martínez, "A group decision method based on prospect theory for emergency situations," Information Sciences, vol. 418-419, pp. 119-135, 2017.

[2] P. Ji, H.-Y. Zhang, and J.-Q. Wang, "A projection-based outranking method with multihesitant fuzzy linguistic term sets for hotel location selection," Cognitive Computation, vol. 10, no. 5, pp. 737-751, 2018.

[3] Z.-P. Tian, J. Wang, J.-Q. Wang, and H.-Y. Zhang, "A likelihood-based qualitative flexible approach with hesitant fuzzy linguistic information," Cognitive Computation, vol. 8, no. 4, pp. 670-683, 2016.

[4] P. Liu and X. Zhang, "A novel picture fuzzy linguistic aggregation operator and its application to group decision making," Cognitive Computation, vol. 10, no. 2, pp. 242-259, 2018.

[5] J. Hu, L. Pan, and X. Chen, "An interval neutrosophic projection-based VIKOR method for selecting doctors," Cognitive Computation, vol. 9, no. 6, pp. 801-816, 2017.

[6] J.-Q. Wang, Y.-X. Cao, and H.-Y. Zhang, "Multicriteria decision making method based on distance measure and
Choquet integral for linguistic Z-numbers," Cognitive Computation, vol. 9, no. 6, pp. 827-842, 2017.

[7] B. Farhadinia and Z. Xu, "Distance and aggregation-based methodologies for hesitant fuzzy decision making," Cognitive Computation, vol. 9, no. 1, pp. 81-94, 2017.

[8] F. Meng, C. Wang, and X. Chen, "Linguistic interval hesitant fuzzy sets and their application in decision making," Cognitive Computation, vol. 8, no. 1, pp. 52-68, 2016.

[9] H.-Y. Zhang, P. Ji, J.-Q. Wang, and X.-H. Chen, "A neutrosophic normal cloud and its application in decision making," Cognitive Computation, vol. 8, no. 4, pp. 649-669, 2016.

[10] X.-H. Xu, Z.-J. Du, and X.-H. Chen, "Consensus model for multicriteria large group emergency decision making considering non-cooperative behaviors and minority opinions," Decision Support Systems, vol. 79, pp. 150-160, 2015.

[11] Y. Liu, Z.-P. Fan, and Y. Zhang, "Risk decision analysis in emergency response: a method based on cumulative prospect theory," Computers \& Operations Research, vol. 42, pp. 75-82, 2014.

[12] $\mathrm{Z} . \mathrm{Wu}$ and $\mathrm{J} . \mathrm{Xu}$, "A consensus model for large scale group decision making with hesitant fuzzy information and changeable clusters," Information Fusion, vol. 41, pp. 217-231, 2018.

[13] A. Pagano, I. Pluchinotta, R. Giordano, A. B. Petrangeli, U. Fratino, and M. Vurro, "Dealing with uncertainty in decision making for drinking water supply systems exposed to extreme events," Water Resources Management, vol. 32, no. 6, pp. 2131-2145, 2018.

[14] B. Sun, W. Ma, B. Li, and X. Li, "Three-way decisions approach to multiple attribute group decision making with linguistic information-based decision-theoretic rough fuzzy set," International Journal of Approximate Reasoning, vol. 93, pp. 424-442, 2018.

[15] S. Bingzhen and M. Weimin, "An approach to evaluation of emergency plans for unconventional emergency events based on soft fuzzy rough set," Kybernetes, vol. 45, no. 3, pp. 461-473, 2016.

[16] C.-G. Cai, X.-H. Xu, P. Wang, and X.-H. Chen, "A multistage conflict style large group emergency decision making method," Soft Computing, vol. 21, no. 19, pp. 5765-5778, 2017.

[17] J. He, C. Feng, D. Hu, and L. Liang, "A decision model for emergency warehouse location based on a novel stochastic mcda method: evidence from China," Mathematical Problems in Engineering, vol. 2017, Article ID 7804781, 10 pages, 2017.

[18] F. Jin, Z. Ni, H. Chen, and Y. Li, "Approaches to decision making with linguistic preference relations based on additive consistency," Applied Soft Computing, vol. 49, pp. 71-80, 2016.

[19] Y. Xu, W. Zhang, and H. Wang, "A conflict-eliminating approach for emergency group decision of unconventional incidents," Knowledge-Based Systems, vol. 83, pp. 92-104, 2015.

[20] Y. Xu, F. Ma, W. Xu, and H. Wang, "An incomplete multigranular linguistic model and its application in emergency decision of unconventional outburst incidents," Journal of Intelligent \& Fuzzy Systems, vol. 29, no. 2, pp. 619-633, 2015.

[21] B. Liu, Y. Shen, X. Chen, H. Sun, and Y. Chen, "A complex multiattribute large group PLS decision making method in the interval-valued intuitionistic fuzzy environment," Applied Mathematical Modelling, vol. 38, no. 17-18, pp. 4512-4527, 2014.

[22] B. Liu, Y. Shen, W. Zhang, X. Chen, and X. Wang, "An interval-valued intuitionistic fuzzy principal component analysis model-based method for complex multiattribute large 
group decision making," European Journal of Operational Research, vol. 245, no. 1, pp. 209-225, 2015.

[23] L. Zhang, Y. Wang, and X. Zhao, "A new emergency decision support methodology based on multisource knowledge in 2tuple linguistic model," Knowledge-Based Systems, vol. 144, pp. 77-87, 2018.

[24] S. Xian, J. Chai, and H. Guo, "Linguistic-induced ordered weighted averaging operator for multiple attribute group decision making," International Journal of Intelligent Systems, vol. 34, no. 2, pp. 271-296, 2019.

[25] J. H. P. Paelinck, "Qualiflex: a flexible multiple criteria method," Economics Letters, vol. 1, no. 3, pp. 193-197, 1978.

[26] A. Isik and E. Adali, "The qualiflex method for the insurance company selection problem," European Scientific Journal, vol. 12, no. 10, pp. 348-358, 2016.

[27] X.-F. Ding, L. Zhang, and H.-C. Liu, "Emergency decision making with extended axiomatic design approach under picture fuzzy environment," Expert Systems, vol. 37, no. 2, 2020.

[28] X.-F. Ding and H.-C. Liu, "An extended prospect theoryVIKOR approach for emergency decision making with 2dimension uncertain linguistic information," Soft Computing, vol. 23, no. 22, pp. 12139-12150, 2019.

[29] X.-F. Ding and H.-C. Liu, "A new approach for emergency decision making based on zero-sum game with Pythagorean fuzzy uncertain linguistic variables," International Journal of Intelligent Systems, vol. 34, no. 7, pp. 1667-1684, 2019.

[30] M.-Y. Li and P.-P. Cao, "Extended TODIM method for multiattribute risk decision making problems in emergency response," Computers \& Industrial Engineering, vol. 135, pp. 1286-1293, 2019.

[31] X. Peng, R. Krishankumar, and K. S. Ravichandran, "Generalized orthopair fuzzy weighted distance-based approximation (WDBA) algorithm in emergency decision making," International Journal of Intelligent Systems, vol. 34, no. 10, pp. 2364-2402, 2019.

[32] J. Gao, Z. Xu, Z. Liang, and H. Liao, "Expected consistencybased emergency decision making with incomplete probabilistic linguistic preference relations," Knowledge-Based Systems, vol. 176, pp. 15-28, 2019.

[33] P. Li and C. Wei, "An emergency decision making method based on D-S evidence theory for probabilistic linguistic term sets," International Journal of Disaster Risk Reduction, vol. 37, p. 101178, 2019.

[34] Y. Song and G. Li, "Consensus constructing in large scale group decision making with multigranular probabilistic 2tuple fuzzy linguistic preference relations," IEEE Access, vol. 7, pp. 56947-56959, 2019.

[35] X. Xu, L. Wang, X. Chen, and B. Liu, "Large group emergency decision making method with linguistic risk appetites based on criteria mining," Knowledge-Based Systems, vol. 182, pp. 1-13, 2019.

[36] X. Xu, Y. Huang, and K. Chen, "Method for large group emergency decision making with complex preferences based on emergency similarity and interval consistency," Natural Hazards, vol. 97, no. 1, pp. 45-64, 2019.

[37] X.-H. Xu, Z.-J. Du, X.-H. Chen, and C.-G. Cai, "Confidence consensus-based model for large scale group decision making: a novel approach to managing noncooperative behaviors," Information Sciences, vol. 477, pp. 410-427, 2019.

[38] X.-H. Xu, X.-Y. Zhong, X.-H. Chen, and Y.-J. Zhou, "A dynamical consensus method based on exit-delegation mechanism for large group emergency decision making," Knowledge-Based Systems, vol. 86, pp. 237-249, 2015.
[39] L. A. Zadeh, "A note on Z-numbers," Information Sciences, vol. 181, no. 14, pp. 2923-2932, 2011.

[40] L. A. Zadeh, "The concept of a linguistic variable and its application to approximate reasoning-I," Information Sciences, vol. 8, no. 3, pp. 199-249, 1975.

[41] D. B. Liu, Uncertainty Theory, Springer Berlin Heidelberg, Heidelberg, Germany, 2007.

[42] X. Zhang, "Multicriteria Pythagorean fuzzy decision analysis: a hierarchical QUALIFLEX approach with the closeness index-based ranking methods," Information Sciences, vol. 330, pp. 104-124, 2016.

[43] T.-Y. Chen, "Interval-valued intuitionistic fuzzy QUALIFLEX method with a likelihood-based comparison approach for multiple criteria decision analysis," Information Sciences, vol. 261, pp. 149-169, 2014.

[44] J.-J. Peng, J.-Q. Wang, and W.-E. Yang, "A multivalued neutrosophic qualitative flexible approach based on likelihood for multicriteria decision making problems," International Journal of Systems Science, vol. 48, no. 2, pp. 425-435, 2017.

[45] J.-Q. Wang, J.-J. Kuang, J. Wang, and H.-Y. Zhang, "An extended outranking approach to rough stochastic multicriteria decision making problems," Cognitive Computation, vol. 8, no. 6, pp. 1144-1160, 2016.

[46] $\mathrm{X} . \mathrm{Xu}$ and $\mathrm{Y} . \mathrm{Yu}$, "Incomplete information complement method based on the hesitation of experts in large group emergency decision making," Information and Control, vol. 48, no. 6, pp. 678-686, 2019.

[47] P. Wang, X. Xu, and S. Huang, "An improved consensusbased model for large group decision making problems considering experts with linguistic weighted information," Group Decision and Negotiation, vol. 28, no. 3, pp. 619-640, 2019. 